\title{
Colchicine in the treatment of refractory aphthous ulcerations: Review of the literature and two case reports
}

\author{
MIHAELA PAULA TOADER ${ }^{1}$, IRINA MIHAELA ESANU ${ }^{2}$, TATIANA TARANU ${ }^{1}$, \\ MADALINA MOCANU ${ }^{1}$ and STEFAN TOADER ${ }^{3}$ \\ Departments of ${ }^{1}$ Oral Dermatology, ${ }^{2}$ Internal Medicine, \\ ${ }^{3}$ Pathophysiology, 'Grigore T. Popa' University of Medicine and Pharmacy, 70015 Iasi, Romania
}

Received October 16, 2020; Accepted November 16, 2020

DOI: $10.3892 / \mathrm{etm} .2021 .9712$

\begin{abstract}
Colchicine has been known and used for over a millennium for its anti-inflammatory properties, being the treatment of choice for gout and familial Mediterranean fever. A tricyclic alkaloid extracted from the herbaceous plant, Colchicum autumnale, colchicine blocks multiple inflammatory pathways, and has antimitotic and antifibrotic action. Although there are insufficient data on the beneficial mechanism of action, colchicine is a widely used treatment in dermatology, one of the morbid conditions with more evidence of efficacy being recurrent aphthous stomatitis (RAS), a disorder with incompletely known etiopathogenesis and, consequently, with poorly defined treatment. Colchicine is considered as the first therapeutic line in complex or severe aphthoses, significantly relieving pain, decreasing the number of lesions, increasing the free interval between eruptive episodes, without inducing noticeable side effects. We examined the treatment efficacy of colchicine in two cases of chronic, severe RAS, evolving in different morbid contexts, who did not respond to other therapeutic measures. The two cases presented with recurrent aphthous stomatitis with herpetiform aphthae; one patient with Turner syndrome and one patient with major Sutton ulcers.
\end{abstract}

\section{Introduction}

Colchicine is a traditional natural remedy known for more than a millennium for its anti-inflammatory properties. Biochemically, it is a toxic protoaloalkaloid from the group of tropolon derivatives, that was extracted in 1819 from the bulb of the autumn crocus (Colchicum autumnale), and in 1833 was named colchicine by the German pharmacist and

Correspondence to: Dr Madalina Mocanu, Department of Oral Dermatology, 'Grigore T. Popa' University of Medicine and Pharmacy, 16 University, 70015 Iasi, Romania

E-mail: drmadalinamocanu@yahoo.com

Key words: colchicine, herpetiform aphthae, Sutton ulcers, refractory aphthous stomatitis, inflammation, anti-inflammatory drugs chemist Philipp Lorenz Geiger (1-3). The pure crystallized form was obtained by the French pharmacist Alfred Houde (1). The current drug is the same purified natural substance. The mechanism of action is still a research topic today. In the years 1950-1960, the main cellular target of colchicine action, the cytoskeleton, was identified. Microtubules are major constituents of the cytoskeleton with a role in cell dynamics, maintaining cell shape through resistance to compression, intracellular transport and cell division (3-7). Colchicine binds to tubulin heterodimers, constituents of protofilaments in the structure of microtubules, to form dimer-colchicine complexes that attach to the end of microtubules causing alterations in their conformation resulting in altered cell function (3-7). Its therapeutic action is attributed to the inhibition of neutrophil chemotaxis, their adhesion and recruitment in inflammatory lesions as colchicine is more concentrated in leukocytes than in plasma (3,4,7-9). Colchicine also suppresses the production of superoxide by neutrophils and reduces oxidative stress by decreasing the influx of calcium ions $\left(\mathrm{Ca}^{2+}\right)$ into neutrophils $(10,11)$. Other confirmed effects include modulation of hepatic macrophage secretion of tumor necrosis factor (TNF) $\alpha$, inhibition of inflammatory cytokine production [interleukin (IL)-1 1 , interferon (IFN) $\gamma$, IL-8, IL-6], promoting dendritic cell maturation and stimulating the presentation of naive $\mathrm{CD} 4^{+}$ lymphocyte antigens, inhibiting vascular endothelial growth factor (VEGF) and endothelial proliferation (3-7,11).

Apart from the anti-inflammatory effect, colchicine also has antifibrotic and cardiovascular protective effects blocking autoinflammatory pathways, including NLRP3 and IL-1 $(3,12)$. Some pathological conditions such as gout or rheumatoid arthritis are associated with high cardiovascular risk due to systemic inflammation. Colchicine is an immune-modulatory agent able to reduce cardiovascular risk for these patients considering inflammation an important component for the development of heart attacks or strokes (13).

After oral administration, colchicine is absorbed in the jejunum and ileum, is metabolized in the liver by the cytochrome P450 (CYP450) enzyme and excreted mainly hepatobiliary and, to a lesser extent, renally $(3,4)$. Drugs inhibiting CYP450 or P-glycoprotein (intracellular transporter molecule important for colchicine absorption and pharmacokinetics), such as erythromycin, clarithromycin, fluconazole, itraconazole, calcium channel blockers (diltiazem, verapamil), 
cyclosporine, tacrolimus or statins enhance the pharmacological effects of colchicine and increase the risk of its toxic effects (11). The official prescribing guidelines for colchicine therefore recommend dose adjustments for patients taking these medications. Colchicine dose reduction is also recommended for patients with severe renal impairment, including patients on hemodialysis, as well as for patients with severe hepatic impairment (14).

Adverse effects are mainly gastrointestinal and are reversible at dose reduction. They consist of abdominal pain, diarrhea, nausea, vomiting and occur in $5-10 \%$ of cases $(6,7)$. Increased levels of serum transaminases, myotoxicity and alopecia are rarely encountered (15-18). Rarer acute adverse effects include myopathy, rhabdomyolysis and myelosuppression. A colchicine neuromyopathy may occur with chronic daily use, particularly in patients whose dose has not been appropriately adjusted for renal disease. Symptoms of colchicine toxicity usually resolve within 1 week to several months of discontinuing the drug (6). The classic therapeutic indications of colchicine are gout and familial Mediterranean fever, as well as its complications (amyloidosis).

Since 2009, colchicine has been approved by the Food and Drug Administration for use in rheumatology, immunology, cardiology, oncology, dermatology (17). The use of colchicine has been shown to be beneficial for the treatment of rheumatic diseases, pericarditis, coronary heart disease, atherosclerosis, and has been attempted in various dermatological diseases, orally or topically, with variable efficacy $(1,3,5,6)$. Severe aphthosis is one of the most documented indications for colchicine treatment with beneficial effects of oral colchicine being reported in case studies, case series and less often, in clinical trials (17-25). We present 2 cases of chronic recurrent aphthous stomatitis (RAS), that represented a diagnostic and therapeutic challenge, as consulted in the Dermatology Clinic of the Railways University Hospital in Iasi, Romania.

\section{Case 1}

A 23-year-old patient, with Turner syndrome treated with somatotropin and, from the age of 13 with estro-progestins (Zoely), presented with multiple extremely painful oral herpetiform exulcerations, with a tendency to group in plaques with a yellowish-gray fibrinous base, persistent for approximately 10 days (Fig. 1).

Patient interrogation revealed the onset of oral lesions 4 years prior to presentation, with an episodic, recurrent course and difficult healing in 2 to 3 weeks, under various topical treatments with analgesics, borax glycerin and corticosteroids. The clinical context (numerous miliary exulcerations located on the non-keratinized labial and lingual mucosa, extremely painful and recurrent, in a female patient under chronic estrogen-progestative substitution treatment) led to the diagnosis of RAS with herpetiform aphthae.

Hematological and biochemical investigations, including dosing of ferritin, folate, zinc, vitamin B12, revealed normal data. Treatment with colchicine $1 \mathrm{mg} / \mathrm{day}$, after 3 days of testing digestive tolerance with $0.5 \mathrm{mg} /$ day, in combination with topical dexamethasone and hyaluronic acid was initiated. The evolution of the lesions over a follow-up period of 10 months, under maintenance treatment with colchicine
$0.5 \mathrm{mg} / \mathrm{day}$, was towards faster healing, on average in 10 days. A greater interval of 2 months between recurrent episodes was also achieved. There were no reported side effects.

\section{Case 2}

A non-smoker 67-year-old patient, with no notable personal medical history, except for pulmonary emphysema, presented for consultation for extremely painful, clearly delimited oval ulcerations with a large transverse axis of approximately $2 \mathrm{~cm}$, with intense erythematous halo and yellowish-gray base, localized on the soft palate and buccal mucosa, with recurrent episodes for 8 years (Fig. 2A).

He was repeatedly evaluated over time by several specialists (ENT, infectious disease, gastroenterology, oral medicine, dermatology). Infectious disease (negative anti-HIV1 and 2 antibodies, AgHbs absent), autoimmune bullous dermatosis (negative anti-desmoglein 3 antibodies), and a neoplastic process (repeated biopsies in 2012, 2013, 2015, with nonspecific inflammatory changes) were excluded. The lesions only partially responded to topical treatments with corticosteroids, lidocaine, oral corticosteroids and dapsone (Fig. 2B). Thus, treatment with colchicine $1 \mathrm{mg} /$ day in combination with pentoxifylline $400 \mathrm{mg} \times 2 /$ day and topical suspension with metronidazole, dexamethasone and nystatin was initiated. The lesions healed significantly in approximately 6 weeks. Treatment with a maintenance dose of colchicine $(0.5 \mathrm{mg} /$ day $)$ was continued for the next 4 months, and was well tolerated with no recurrent episodes during this time (Fig. 2C).

\section{Discussion}

Efficacious results of colchicine treatment have been reported in several dermatologic conditions, such as chronic urticaria unresponsive to antihistamines, urticarial vasculitis, forms of cutaneous vasculitis (hypocomplementemic urticarial, leukocytoclastic, nodular, necrotic vasculitis, Henoch-Schonlein purpura), palmo-plantar pustular psoriasis (applied as a hydrophilic ointment with colchicine 1\%), pyoderma gangrenosum associated or not with inflammatory bowel disease, Sweet syndrome, subcorneal pustulosis, acquired bullous epidermolysis with limited skin lesions, benign mucosal pemphigoid, Behcet's disease, actinic keratosis (applied as a hydrophilic gel with colchicine $1 \%$ ) or granuloma annulare $(3,26-34)$. Less satisfactory results have been obtained for hidradenitis suppurativa, acne vulgaris, dermatitis herpetiformis, linear IgA dermatosis, scleroderma and psoriasis vulgaris $(3,35,36)$.

Recurrent aphthous stomatitis (RAS) is a recurrent ulcerative stomatitis with an estimated prevalence between 2 and $10 \%$, with an incompletely elucidated etiopathogenesis and, consequently, with poorly defined treatment (37). In developed countries, the incidence in the general population reaches $20 \%$, mainly affecting young adults (38). The pathophysiological substrate consists of an antigenic stimulation of oral mucosal keratinocytes in predisposed individuals, followed by the secretion of proinflammatory cytokines (especially IL-2, TNF $\alpha$ ) and the consequent expression of class I major histocompatibility complex antigens (MHC). MHC class I antigen-expressing cells become targets of cytotoxic $\mathrm{T}$ lymphocytes $(39,40)$. The inflammatory process, resulting in 


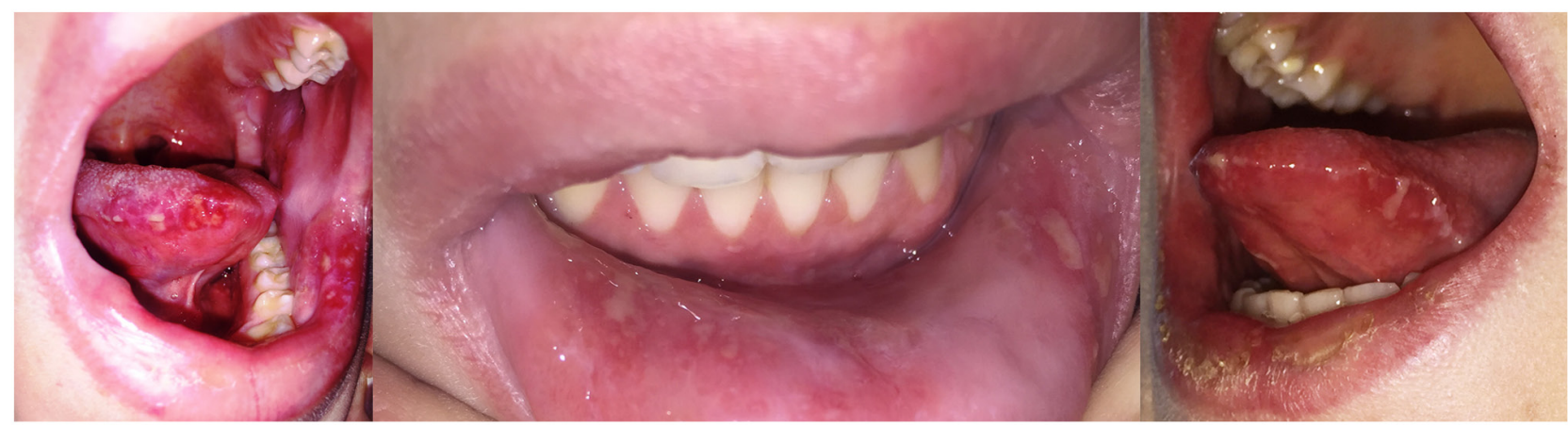

Figure 1. Multiple millimetric exulcerations grouped in erosive patches with micropolicyclic contour on the labial and non-keratinized lingual mucosa.
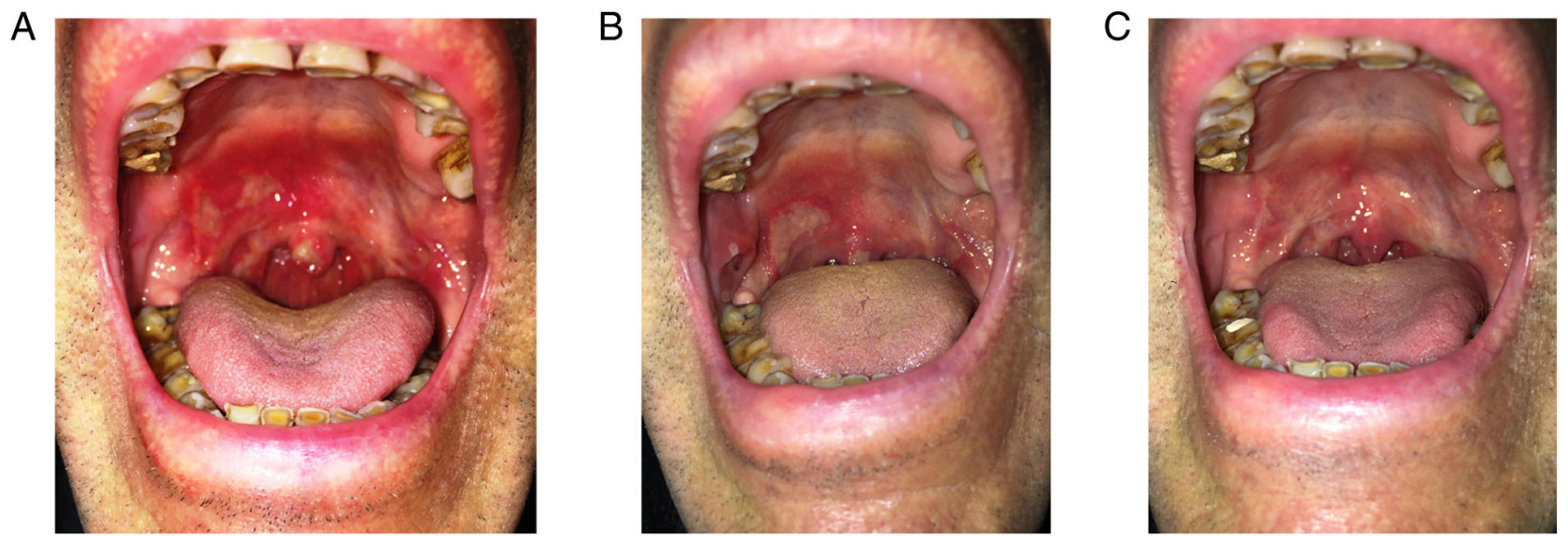

Figure 2. (A) Initial presentation with large ulcers on the soft palate and buccal mucosa. (B) Clinical aspect after treatment with dapsone for 6 weeks. (C) Clinical aspects after treatment with colchicine for 6 weeks (almost complete epithelization of oral ulcers).

variable epithelial necrosis depending on its histopathological site, is the consequence of an aberrant immune response, influenced by an abnormal oral flora (4).

The three modes of clinical expression of the disorder are common aphthae (a few round or oval exulcerations with an average diameter of 2-4 $\mathrm{mm}$, with a gray-yellow base and a characteristic carmine-red areola, with self-limited evolution of approximately 7-10 days), herpetiform aphthae, the rarest (numerous yellowish, millimetric exulcerations, with a tendency to coalesce in erosive patches with micropolycyclicontour, evolving for approximately two weeks) and major aphthae (Sutton's ulcers or periadenitis mucosa necrotica recurrens), the most severe clinical form (crateriform ulcers, with a diameter between 1 and $3 \mathrm{~cm}$, often solitary, accompanied by satellite adenopathy, with difficult healing for 1-2 months with sometimes mutilating scars) $(8,39,40)$. The common clinical features of these ulcerations are intense pain, location on non-keratinized areas of the oral mucosa, self-limiting character and recurrences, either spontaneous or correlated with triggering factors. These factors may be local (e.g. oral trauma, contact hypersensitivity, sodium lauryl sulfate), nutritional deficiencies (iron, vitamin B12, folic acid), medications (angiotensin converting enzyme inhibitors, gold salts, phenobarbital, diclofenac, piroxicam), inflammatory bowel disease (gluten-sensitive enteropathy, Crohn's disease, ulcerative colitis), certain foods (tomatoes, nuts, cocoa, dairy, spices), or a hormonal context with progesterone deficiency in females. RAS is also correlated with a genetic predisposition $(3,8,39)$.

The treatment of RAS is a challenge. In the absence of a clear etiopathogenesis, various topical and systemic therapies have been attempted over time, with the aim to reduce pain, shorten the duration of recurrent episodes, to distance them in time, and in order to improve the quality of life of these patients (40). Severe aphthoses, with very painful lesions accompanied by functional signs (pain when speaking, chewing, swallowing), with frequent recurrences and significant psycho-social impact, raise the issue of systemic therapy. Prednisone, thalidomide, cyclosporine, azathioprine, methotrexate, dapsone, pentoxifylline, and colchicine have been administered. The use of colchicine in the treatment of RAS is well documented. The mechanism by which colchicine positively influences RAS lesions could be essentially explained by inhibiting leukocyte chemotaxis and mobilization, their lysosomal degranulation, by modulating the interaction between leukocytes and vascular endothelium, and by decreasing the production of proinflammatory cytokines, including IL-6 and IL-1ß $(19,41)$. Studies support the effectiveness of colchicine in RAS, both as a monotherapy and in combination with other systemic therapies, as an episode treatment and as a maintenance or prophylactic treatment. The first large retrospective study (1986-2000), on 54 immunocompetent patients with RAS followed on average for 4.7 years, concluded that the administration of colchicine at doses of $1-1.5 \mathrm{mg} / \mathrm{day}$ for at 
least 3 months had beneficial effects on episode frequency, pain intensity and RAS impact on quality of life in $63 \%$ of patients, $37 \%$ of whom maintained results for 5 years (41). Very beneficial effects on reducing pain and injury were reported in a series of 20 patients with severe aphthosis, treated with colchicine $0.5 \mathrm{mg}$ three times per day (20). The mean weekly number of aphthae and pain score were assessed in patients treated continuously with colchicine compared to those treated only 2 months after 2 previous months without treatment. This 4-month study highlighted the obvious prophylactic benefit of uninterrupted treatment with colchicine (21). Persistent significant remissions for 3-5 years were also reported in 2 of a series of 3 patients with severe aphthosis treated with colchicine. Administration of colchicine at a dose of $0.5 \mathrm{mg}$ three times per day, 3 consecutive days/week had a similar efficacy to that of prednisolone administered at a dose of $5 \mathrm{mg} /$ day and to combination therapy with levamisole $50 \mathrm{mg} /$ day and prednisolone $5 \mathrm{mg} /$ day in a study on 50 patients with RAS followed for 3 weeks (22). Colchicine can also be used as a prophylactic treatment. Doses with a prophylactic effect of 0.5-1.8 mg/day have been reported in a study on 9 children with PFAPA (periodic fever, aphthous stomatitis, pharyngitis, cervical adenitis) and were found to significantly increase the free interval between episodes of oral ulceration (23). Another randomized controlled open label study (18 patients aged 4-11 years) over a period of 6 months, followed the number of active episodes in two groups: One treated for 3 months after 3 months of surveillance and one control. The number of episodes were similar in the first 3 months without treatment in both groups. The number of patients treated during the follow-up 3 months was significantly lower compared to the control and compared to the first 3 months of the study - the authors emphasizing the definite prophylactic role of colchicine. Daily doses of $0.5-1.5 \mathrm{mg}$ of colchicine alone or in combination with dapsone (75-100 $\mathrm{mg}$ /day) were also shown to be beneficial in another study of 55 patients with severe aphthosis monitored between 1998 and 2007, 80\% of them with a substantial response $(24,25)$.

Considering the clinical context of the disease, with severe episodes, only partially responsive to classic therapies, the two presented cases are part of the group of complex aphthoses. The response was favorable to colchicine, starting with a dose of $1 \mathrm{mg}$ /day until significant remission was obtained, and continuing with a maintenance dose of $0.5 \mathrm{mg} /$ day for several months of follow-up (10 and 4 months, respectively, in our cases), recording only two mild recurrences in the patient with herpetiform aphthae and no recurrence in the patient with Sutton's ulcers. Colchicine was associated with pentoxifylline in our second patient, considering literature reports of its beneficial effects in reducing the severity and frequency of aphthous ulcer episodes. Pentoxifylline inhibits the production of $\mathrm{TNF} \alpha$ and reduces the migration of neutrophils, but its specific action in aphthous stomatitis is still unclear (42). There were no side effects in any of our patients during the follow-up period.

In conclusion, inhibition of multiple inflammatory pathways and modulation of the innate immune response are the main attributes of colchicine exploited in the treatment of several dermatoses, including RAS. Case studies, series of patients and clinical trials, although few, provide evidence of the efficacy of colchicine (level of evidence III) in severe aphthosis, refractory to classical therapies with topical or systemic corticosteroids, pentoxifylline, and cyclosporine. There is no consensus on the ideal therapeutic regimen for colchicine in RAS.

Therapeutic doses of $0.5-1.5 \mathrm{mg} /$ day are usually free of noticeable side effects, even after 6-9 months of treatment, provided that drug interactions are avoided and doses are adjusted in patients with hepatic or renal impairment. The choice of treatment with colchicine in severe aphthosis should take into account the severity of the lesions, their chronic nature, the lack of therapeutic efficacy of other medications and the context of the patient morbidity. The mechanism of action underlying the efficacy of colchicine in various dermatoses, as well as the optimal therapeutic regimen, including RAS, require further extensive research.

\section{Acknowledgements}

Not applicable.

\section{Funding}

No funding was received.

\section{Availability of data and materials}

The data that support the findings of this study are available from the archives of the Railways University Hospital Iasi, (Iasi, Romania), but restrictions apply to the availability of these data which are not publicly available. Data are, however, available from the authors upon reasonable request and with permission from the Railways University Hospital Iasi.

\section{Author's contributions}

TT conceived and supervised the study. MPT, IME and ST analyzed the data. MPT, TT, MM, IME and ST contributed to data acquisition and interpretation and wrote the manuscript. All authors contributed equally to acquisition, analysis and systematization of data, manuscript writing and critical revision of it for important intellectual content. All authors reviewed the results and read and approved the final version of the manuscript.

\section{Ethics approval and consent to participate}

The Research Ethics Committee of the Railways University Hospital Iasi (Iasi, Romania) affiliated with 'Grigore T. Popa' University of Medicine and Pharmacy approved the current study.

\section{Patient consent for publication}

Informed written consent was obtained from both patients for publication of the case reports.

\section{Competing interests}

The authors declare that they have no competing interests. 


\section{References}

1. Karamanou M, Tsoucalas G, Pantos K and Androutsos G: Isolating colchicine in 19th century, an old drug revisited. Curr Pharm Des 24: 654-658, 2018

2. Nerlekar N, Beale A and Harper RW: Colchicine-a short history of an ancient drug. Med J Aust 201: 687-688, 2014.

3. Robinson KP and Chan JJ: Colchicine in dermatology: A review. Australas J Dermatol 59: 278-285, 2018.

4. Leung YY, Yao Hui LL and Kraus VB: Colchicine-Update on mechanisms of action and therapeutic uses. Semin Arthritis Rheum 45: 341-350, 2015.

5. Slobodnick A, Shah B, Pillinger MH and Krasnokutsky S: Colchicine: Old and new. Am J Med 128: 461-470, 2015.

6. Slobodnick A, Shah B, Krasnokutsky S and Pillinger MH: Update on colchicine, 2017. Rheumatology (Oxford) 57 (Suppl 1): i4-i11, 2018.

7. Paschke S, Weidner AF, Paust T, Marti O, Beil M and Ben-Chetrit: Technical advance: Inhibition of neutrophil chemotaxis by colchicine is modulated through viscoelastic properties of subcellular compartments. J Leukoc Biol 94: 1091-1096, 2013.

8. Peter T, Cherian D and Peter T: Recurrent aphtous stomatitis: Mystery unravelled. J Clin Exp Res 2: 141-145, 2014.

9. Altinor S, Oztürkcan S and Hah MM: The effects of colchicine on neutrophil function in subjects with recurrent aphthous stomatitis. J Eur Acad Dermatol Venereol 17: 469-470, 2003.

10. Chia EW, Grainger R and Harper JL: Colchicine suppresses neutrophil superoxide production in a murine model of gouty arthritis: A rationale for use of low-dose colchicine. $\mathrm{Br}$ J Pharmacol 153: 1288-1295, 2008.

11. Korkmaz S, Erturan I, Nazıroğlu M, Uğuz AC, Cï̆ B and Övey IS: Colchicine modulates oxidative stress in serum and neutrophil of patients with Behçet disease through regulation of $\mathrm{Ca}^{2+}$ release and antioxidant system. J Membr Biol 244: 113-120, 2011.

12. Ozkok A: Cholesterol-embolization syndrome: Current perspectives. Vasc Health Risk Manag 15: 209-220, 2019.

13. Crowson CS, Liao KP, Davis JM III, Solomon DH, Matteson EL, Knutson KL, Hlatky MA and Gabriel SE: Rheumatoid arthritis and cardiovascular disease. Am Heart J 166: 622-628, 2013.

14. Tarakji B, Gazal G, Al-Maweri SA, Azzeghaiby SN and Alaizari N: Guideline for the diagnosis and treatment of recurrent aphthous stomatitis for dental practitioners. J Int Oral Health 7 74-80, 2015.

15. Imazio M, Belli R, Brucato A, Cemin R, Ferrua S, Beqaraj F, Demarie D, Ferro S, FornoD, Maestroni S, et al: Efficacy and safety of colchicine for treatment of multiple recurrences of pericarditis (CORP-2): A multicentre, double-blind, placebo-controlled, randomised trial. Lancet 383: 2232-2237, 2014.

16. Oh DH, Chan SQ and Wilson AM: Myopathy and possible intestinal dysfunction in a patient treated with colchicine and simvastatin. Med J Aus 197: 332-333, 2012.

17. Dasgeb B, Kornreich D, McGuinn K, Okon L, Brownell I and Sackett DL: Colchicine: An ancient drug with novel applications. Br J Dermatol 178: 350-356, 2018.

18. Dalmau J, Alegre M, Domingo P and Alomar A: Major ora aphtous ulceration in HIV-1 infection: Successful response after highly active antiretroviral therapy. J Eur Acad Dermato Venereol 21: 126-127, 2007.

19. Ruah CB, Stram JR and Chasin WD: Treatment of severe recurrent aphthous stomatitis with colchicine. Arch Otolaryngol Head Neck Surg 114: 671-675, 1988.

20. Fontes V, Machet L, Huttenberger B, Lorette G and Vaillant L: Recurrent aphthous stomatitis: Treatment with colchicine. An open trial of 54 cases. Ann Dermatol Venereol 129: 1365-1369, 2002 (In French).

21. Katz J, Langevitz P, Shemer J, Barak S and Livneh A: Prevention of recurrent aphthous stomatitis with colchicine: An open trial. J Am Acad Dermatol 31: 459-461, 1994.
22. Pakfetrat A, Mansourian A, Momen-Heravi F, Delavarian Z, Momen-Beitollahi J, Khalilzadeh O and Basir-Shabestari S: Comparison of colchicine versus prednisolone in recurrent aphthous stomatitis: A double-blind randomized clinical trial. Clin Invest Med 33: E189-E195, 2010.

23. Tasher D, Stein M, Dalal I and Somekh E: Colchicine prophylaxis for frequent periodic fever, aphthous stomatitis, pharyngitis and adenitis episodes. Acta Paediatr 97: 1090-1092, 2008

24. Butbul Aviel Y, Tatour S, Gershoni Baruch R and Brik R Colchicine as a therapeutic option in periodic fever, aphthous stomatitis, pharyngitis, cervical adenitis (PFAPA) syndrome. Semin Arthritis Rheum 45: 471-474, 2016.

25. Lynde CB, Bruce AJ and Rogers RS III: Successful treatment of complex aphthosis with colchicine and dapsone. Arch Dermatol 145: 273-276, 2009.

26. Pho LN, Eliason MJ, Regruto M, Hull CM and Powell DL: Treatment of chronic urticaria with colchicine. J Drugs Dermatol 10: 1423-1428, 2011.

27. Jachiet M, Flageul B, Deroux A, Le Quellec A, Maurier F, Cordoliani F, Godmer P, Abasq C, Astudillo L, Belenotti P, et al: French vasculitis study group: The clinical spectrum and therapeutic management of hypocomplementemic urticarial vasculitis: Data from a French nationwide study of fifty-seven patients. Arthritis Rheumatol 67: 527-534, 2015.

28. Goeser MR, Laniosz V and Wetter DA: A practical approach to the diagnosis, evaluation, and management of cutaneous small-vessel vasculitis. Am J Clin Dermatol 15: 299-306, 2014.

29. Kontochristopoulos GJ, Stavropoulos PG, Gregoriou S and Zakopoulou N: Treatment of pyoderma gangrenosum with low-dose colchicine. Dermatology 209: 233-236, 2004.

30. Maillard H, Leclech C, Peria P, Avenel-Audran M and Verret JL: Colchicine for Sweet's syndrome. A study of 20 cases. $\mathrm{Br}$ J Dermatol 140: 565-566, 1999.

31. Adisen E, Tekin O, Gulekon and Gürer MA: A retrospective analysis of treatment responses of palmoplantar psoriasis in 114 patients. J Eur Acad Dernatol Venereol 23: 814-819, 2009.

32. Pavithran K: Colchicine in the treatment of subcorneal pustular dermatosis. Indian J Dermatol Venereol Leprol 61: 56-57, 1995.

33. Gürcan HM and Ahmed AR: Current concepts in the treatment of epidermolysis bullosa acquisita. Expert Opin Pharmacother 12: 1259-1268, 2011.

34. Chaidemenos G, Sidiropoulos T, Katsioula P and KoussidouEremondi T: Colchicine in the management of mucous membrane pemphigoid. Dermatol Ther 24: 443-445, 2011.

35. van derZee HH and Prens EP: The anti-inflammatory drug colchicine lacks efficacy in hidradenitis suppurativa. Dermatology 223: 169-173, 2011.

36. Schepis C, Siragusa M, Palazzo R and Guerra AP: Failure of colchicine in the treatment of severe acne vulgaris. Acta Derm Venereol 79: 491, 1999.

37. Altenburg A, Abdel-Naser MB, Seeber H, Abdallah M and Zouboulis CC: Practical aspects of management of recurrent aphthous stomatitis. J Eur Acad Dermatol Venereol 21: 1019-1026, 2007

38. Bischoff EW, Uijen A and van der Wel M: Aphthous ulcers. BMJ 339: b2382, 2009.

39. Preeti L, Magesh K, Rajkumar K and Karthik R: Recurrent aphthous stomatitis. J Oral Maxillofac Pathol 15: 252-256, 2011.

40. Altenburg A and Zouboulis CC: Current concepts in the treatment of recurrent aphthous stomatitis. Skin Therapy Lett 13: 1-4, 2008.

41. Mimura MA, Hirota SK, Sugaya NN, Sanches JA Jr and Migliari DA: Systemic treatment in severe cases of recurrent aphthous stomatitis: An open trial. Clinics (Sao Paulo) 64: 193-198, 2009.

42. Abdulrahman HS and Mutaz FF: Therapeutic management of recurrent aphthous stomatitis: A review of the growing knowledge. Ann Int Med Dent Res 2: 1-9, 2016. 